\title{
THE ANGULAR SIZE - REDSHIFT RELATION AS A COSMOLOGICAL TOOL
}

\author{
V.K.Kapahi \\ Tata Institute of Fundamental Research \\ Post Box 1234, Bangalore 560012 \\ India
}

\section{INTRODUCTION}

The angular size - redshift $(\theta-z)$ relation can in principle be used to discriminate between world models because the angular size subtended by a rigid rod is quite a sensitive function of cosmology, specially at $z>0.5$. The test is simpler to apply to objects for which a metric diameter is measured than to objects with isophotal diameters (Sandage 1961). It was first suggested by Hoyle (1958) at the Paris symposium on Radio Astronomy, that the separation between the two lobes of extragalactic radio sources such as Cyg-A, could be used for performing such a test. In an Einstein-de Sitter Universe sources like Cyg-A cannot have angular sizes $<15$ arcsec (the minimum occuring at $z=1.25$ ) whereas in the Steady State Universe their sizes should asymptotically approach a value near 4 arcsec at large redshifts. It was not until the early seventies that the test was actually applied to samples of radio quasars with redshifts of upto 2 (Legg 1970; Miley 1971; Wardle \& Miley 1974). The angular sizes were found to show a large scatter due to a wide distribution of physical sizes and the projection effects associated with the essentially linear radio structures. The upper envelope to the $\theta$-values (which would be expected to show much less scatter) nevertheless appeared to fall off monotonically with increasing $\mathrm{z}$, more or less like the Euclidean relation $\theta \propto z^{-1}$. The $\theta-z$ test thus appeared to be incompatible with the predictions of uniform world models in which the linear sizes of quasars are independent of epoch.

In the Friedmann models of the universe the straightforward interpretation of the $\theta-z$ plots is that linear sizes $(\ell)$ of quasars were systematically smaller at earlier epochs. If a simple epoch dependence of the form $\ell(z)$ $\propto \ell(z=0)(1+z)^{-n}$ is assumed, values of the evolution parameter, $n$, of 1 to 2 are necessary to fit the data. Such evolution can closely mimic an Euclidean relation between $\theta$ and $z$. The evolutionary interpretation was not unique, however, because flux density limited samples of radio sources show a strong correlation between radio luminosity $(P)$ and redshift (the ubiquitous Malmquist bias), so that a possible inverse correlation between $\mathrm{P}$ and $\ell$ cannot be easily distinguished from size evolution (eg. Jackson 1973; Stannard \& Neal 1977; Wardle \& Potash 1977; Hooley et al. 1978; Wills 1979; Saikia 
\& Kulkarni 1979; Masson 1980; Macklin 1982).

In this review I will discuss the observed $\theta-z$ relation for large samples of quasars and radio galaxies that are currently available, and the recent attempts to disentangle the dependence of linear sizes on radio luminosity and redshift. I will also briefly consider the present situation with regard to the angular size - flux density $(\theta$ - S) relation, whose interpretation has been somewhat controversial. The conclusion now seems inescapable that the maximum linear sizes of extragalactic radio sources have undergone fairly strong evolution with cosmic epoch.

I will not discuss here the application of the $\theta-z$ test to clusters of galaxies (Hickson 1977; Bruzual \& Spinrad 1978) which also appears to be strongly affected by evolution of cluster sizes with epoch (see Hickson \& Adams 1979 for a discussion).

\section{THE $\theta-z$ RELATION FOR QUASARS AND RADIO GALAXIES}

The $\theta-z$ plots for 296 quasars and 215 radio galaxies are shown separately in Figures 1 and 2 respectively. The plot for quasars includes essentially all those with known redshift that have a steep high frequency spectral index (generally 1.4 to $5 \mathrm{GHz} ; \alpha>0.5 ; \mathrm{S} \propto \nu^{-\alpha}$ ) and for which the angular sizes have been measured with sufficient angular resolution with aperture synthesis telescopes. Our starting point was the compilation of quasar data by VeronCetty \& Veron (1985). Spectral and structural information was compiled
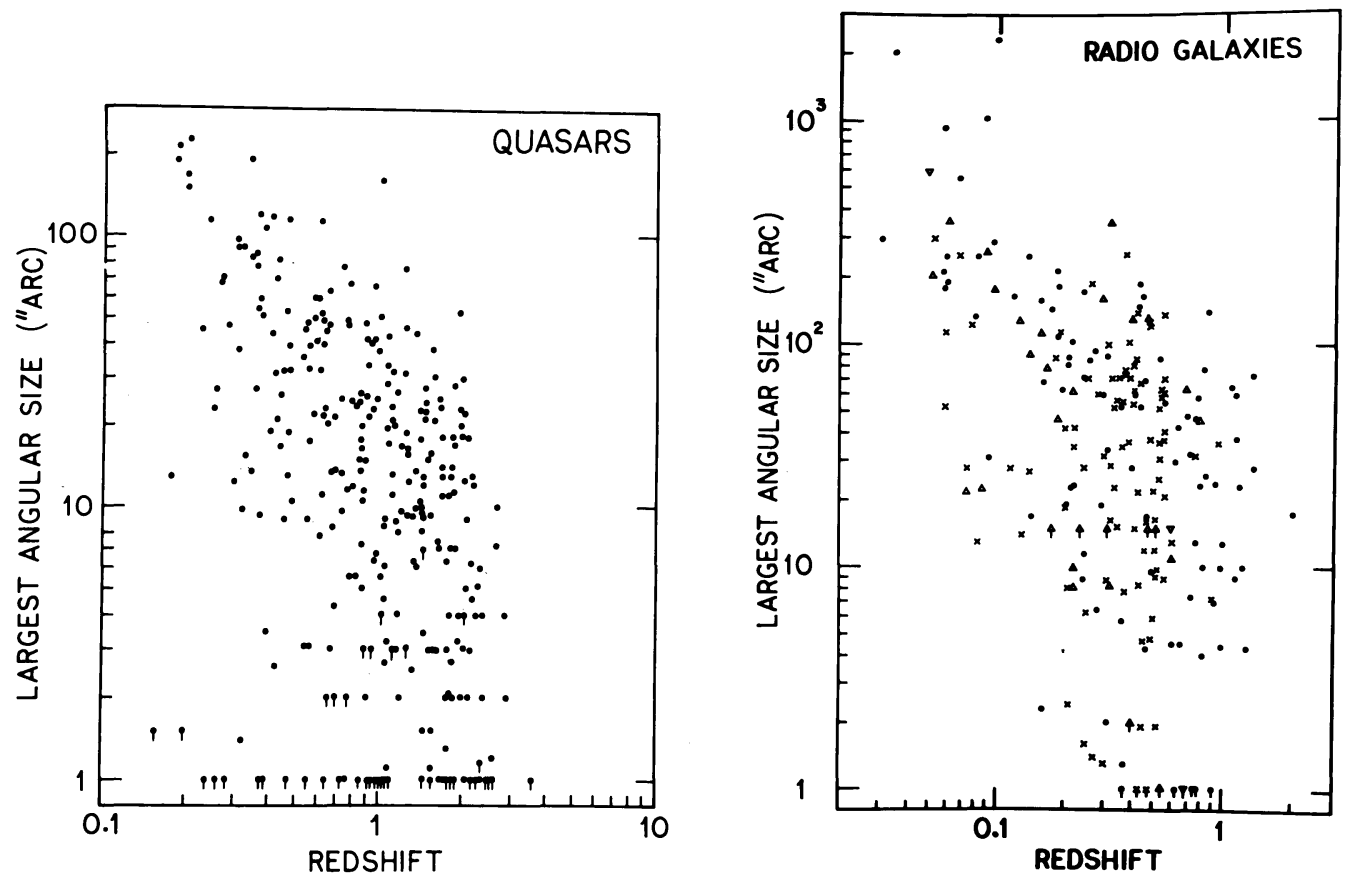

Figs. 1 \& 2. The $\theta-z$ plots of quasars and radio galaxies respectively. 
irom a large number of references. As the number of radio galaxies with large measured redshifts is much smaller than quasars, the galaxy sample was put together as follows. We restricted the search to the following complete samples for which good optical identification and structural information is available; the 3CR sample (Laing et al. 1983); BDFL sample (Bridle et al. 1972); $2.7 \mathrm{GHz}$ northern hemisphere Catalogue (Peacock \& Wall 1981) and the GB/GB2 samples of radio galaxies (Machalski \& Condon 1985). Spectroscopic redshifts are not available for most of the galaxies in the last sample; we have however used the redshifts estimated from their optical magnitudes by Machalski \& Condon. We exclude galaxies with radio spectral indices $<0.5$ and those with estimated radio luminosities $\mathrm{P}(408 \mathrm{MHz}) \leqslant 10^{25} \mathrm{WHz}^{-1}$ $\operatorname{ster}^{-1}\left(\mathrm{H}_{0}=50 \mathrm{~km} \mathrm{~s}^{-1} \mathrm{Npc}^{-1} ; \mathrm{q}_{\mathrm{O}}=1 / 2\right)$ because on the Fanaroff \& Riley (1974) classification most such objects do not have the edge brightened structure typical of quasars.

As can be seen from Figures 1 and 2 there is a large scatter in $\theta$ values at all redshifts, but the upper envelope shows a monotonic decrease of $\theta$ with increasing redshift. The upper envelope is however not a good parameter for quantitative analysis (there is in fact the possibility that one or two objects that strongly influence the estimate of the upper envelope may not have been plotted correctly, either because their redshifts, estimated from just a couple of emission lines, may be grossly in error, or their angular sizes may have been overestimated due to the chance presence of an unrelated radio source in a direction close to their radio axes). We shall therefore use the median angular size $\left(\theta_{m}\right)$ as the more appropriate parameter to characterise the angular sizes. Although the samples we have used are not strictly complete, it is very unlikely that selection or instrumental effects would have introduced a significant systematic dependence of $\theta$ on $z$ of the form apparent in Figures 1 and 2 .

A word about the steep-spectrum compact (SSC) sources. Many of these are unresolved and appear in the plots mostly at high redshifts and are more common among quasars than radio galaxies, as is known from complete surveys at high frequencies (Kapahi 1981; Peacock \& Wall 1982; Kapahi et al. 1986). Their angular sizes do not appear to form a smooth continuation of the distribution of sizes of the more extended sources (particularly apparent in Figures 1 and 2 at lower redshifts). Most of the SSC sources (generally $<20 \mathrm{kPC}$ in extent) are known to show low frequency turnovers in their spectra due to synchrotron self absorption or thermal absorption effects (eg. van Breugel et al. 1984). As they may form a class quite distinct from the extended objects that we are essentially interested in for the $\theta-z$ tests, we shall exclude such objects (all those with linear sizes $<20 \mathrm{kpc}$ ) from consideration, but note that their inclusion only strengthens our conclusions regarding evolution in linear sizes.

The median angular sizes for radio galaxies and quasars in different ranges of $\mathrm{z}$ are shown in Figure 3. There appears to be a very good agreement in the values of $\theta_{\mathrm{m}}$ for radio galaxies and quasars in the region of overlap in redshift. Also, the sizes of radio galaxies at low redshifts show a smooth continuity with those of quasars at higher redshifts. So whatever their other differences, radio galaxies and quasars appear to be indistinguishable as far as the linear sizes of their extended radio structure is concerned. It is also worth noting that the excellent continuity between radio galaxies and quasars, together with the decrease in angular sizes with redshift, provides 


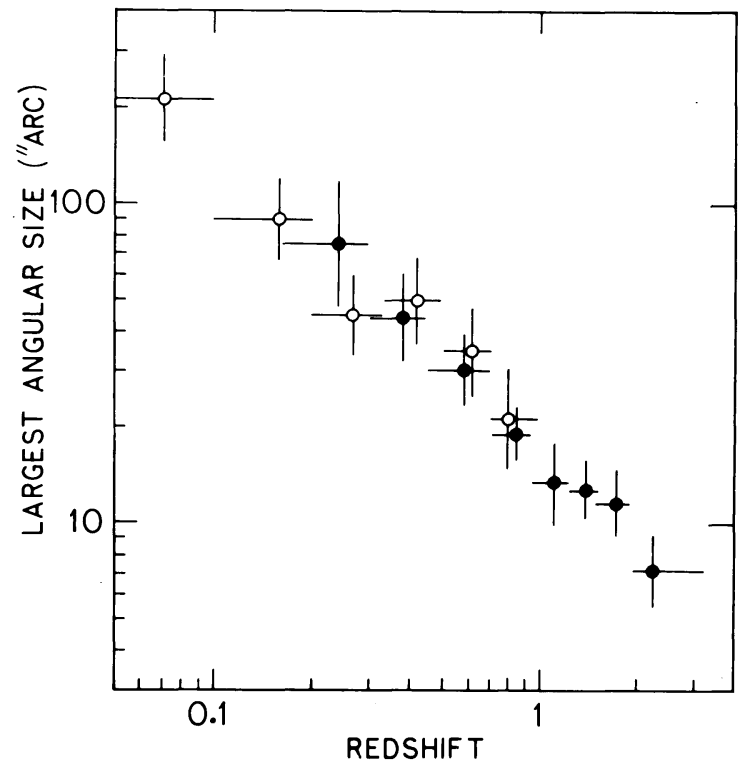

Fig. 3. The median angular size in different ranges of $\mathrm{z}$ for quasars (filled circles) and radio galaxies (unfilled circles).

strong support to the cosmological nature of redshifts. Any non-cosmological interpretation of quasar redshifts must explain these features of Figure 3.

Before comparing the observed $\theta-z$ relation with predictions of different world models we shall first examine the possible dependence of angular sizes on radio luminosity.

\section{DEPENDENCE OF LINEAR SIZES ON RADIO LUMINOSITY}

The strong correlation between $\mathrm{z}$ and $\mathrm{P}$ in the data for quasars due to the Malmquist bias is shown in Fig.4. A similar correlation exists for the radio galaxies. In order to investigate the epoch dependence of linear sizes it is therefore first necessary either to determine the luminosity dependence of linear sizes by comparing the sizes of samples with widely different luminosities at the same redshift or to determine the epoch dependence from samples of similar luminosity at different redshifts. With the availability of deep optical photometry on some samples, only recently has it become possible to carry out such a comparison with reasonable statistical significance (Kapahi 1985, 1986; Eales 1985; Kapahi \& Kulkarni, in preparation). We will review this briefly.

Kapahi (1986) has considered four complete samples of radio galaxies selected from $1.4 \mathrm{GHz}$ surveys to have similar luminosities at different redshifts as follows. i) BDFL; $\mathrm{S}(1.4 \mathrm{GHz}) \geq 2 \mathrm{Jy}$; $0.075<\mathrm{z}<0.2$, ii) GB/GB2; $\mathrm{S}(1.4)$ $\geq 0.55 \mathrm{Jy} ; 0.15<\mathrm{z}<0.4$, iii) GB/GB2; S(1.4) >0.2 Jy; $0.25<\mathrm{z}<0.6$ and iv) LBDS; $\mathrm{S}(1.4)>10 \mathrm{mJy} ; \mathrm{z} \approx 0.8$. The choice of these samples and redshift ranges is based on the availability of good angular size information and the need to have a reasonably large number of sources for statistics. The relati- 


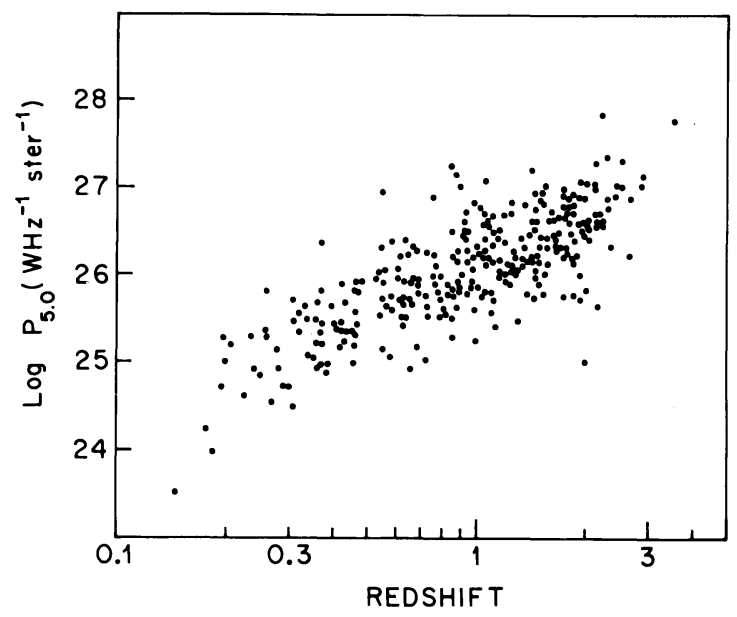

Fig. 4. The correlation between redshift and radio luninosity for quasars.

vely small range of $\mathrm{P}$ covered by the samples is shown in Fig.5. Note that spectroscopic redshifts are known for most of the sources only in the brightest BDFL sample and have been estimated (by Machalski \& Condon 1985) from the optical magnitudes for the GB/GB2 samples. The Leiden Berkeley Deep Survey (LBDS) sample (Windhorst et al. 1985a). consists of all those sources in the mentioned flux density range that are either identified with galaxies of $\mathrm{F}$ magnitude $>22$ or have no optical counterparts (implying $\mathrm{F} \gtrsim 22.75$ ) down to the plate limit (Windhorst et al. 1985b). Most of these are expected to be galaxies at $z \approx 0.8$. The angular sizes of these sources have now been determined with high angular resolution $(\sim 1.2$ arcsec $)$ using the VLA at 21 $\mathrm{cm}$ (Kapahi \& Kulkarni, in preparation). The median angular sizes for these four samples are shown in Figure 7 (using crosses) for comparison with the other data for radio galaxies and quasars. Although there is little direct

Fig. 5. The regions of the $\mathrm{P}-\mathrm{z}$ plane covered by 4 samples of radio galaxies discussed in the text.

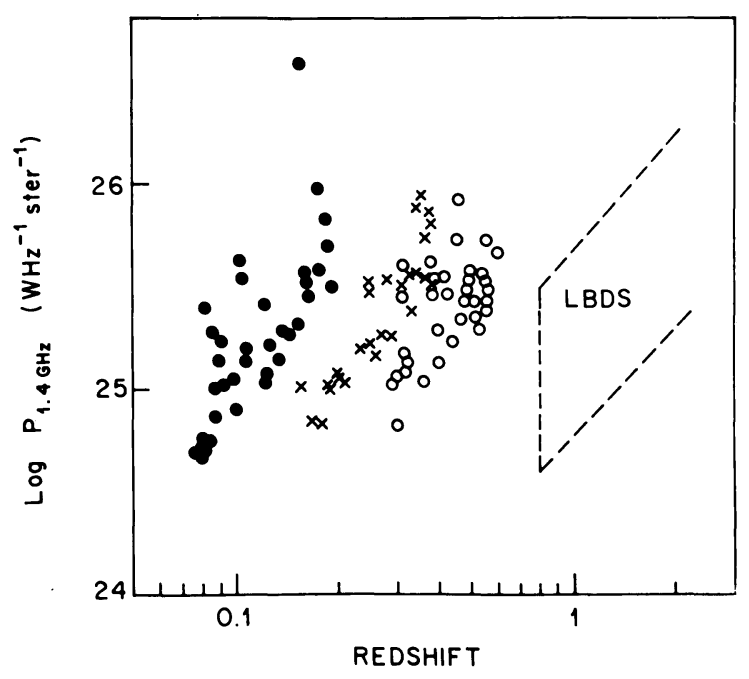


information about the redshifts of sources in the LBDS sample, it seems unlikely that the redshifts for radio galaxies extend much beyond $\sim 2$ or so (Windhorst 1986; Downes et al. 1986). We have assumed a median redshift of 1.25 for this sample but the conclusions are not likely to be seriously affected even if the actual $z_{m}$ is considerably different $(0.8 \leqslant z \leqslant 2)$. Note also that the values of $\theta_{\mathrm{m}}$ for these samples should be directly comparable with the other data in Figure 7 since the number of flat spectrum $(\alpha<0.5)$ sources is negligible ( $<10 \%$; see Figure 6$)$ in all the four samples.

The value of $\theta_{\mathrm{m}}$ for the LBDS sample is seen in Fig.7 to be considerably smaller than for quasars in a comparable redshift range. As the quasars come mostly from much stronger radio surveys than the LBDS, they are of higher radio luminosity. This means that the angular sizes are likely to be directly correlated"with radio luminosity rather than being smaller at higher luminosities. This can be seen more clearly from Figure 6 in which we have compared the linear size distributions in the GB/GB2 samples and in the LBDS sample with complete samples of radio galaxies from the strong source 3CR sample (Laing et al. 1983) in the same redshift ranges. The comparison is thus between sources of different luminosities in three different redshift ranges. The 3CR galaxies compared in Fig.6 with the LBDS sample consist of all those that are either known or estimated (from optical magnitudes) to have $z>0.8$ together with the few sources that still do not have optical counterparts. If these two samples have a similar distribution of redshifts, their median luminosities must differ by about 2 orders of magnitude. There is clearly no evidence in Fig.6 of a $\mathrm{P}-\ell$ anticorrelation at any redshift. The higher luminosity $3 C R$ galaxies in general appear to have

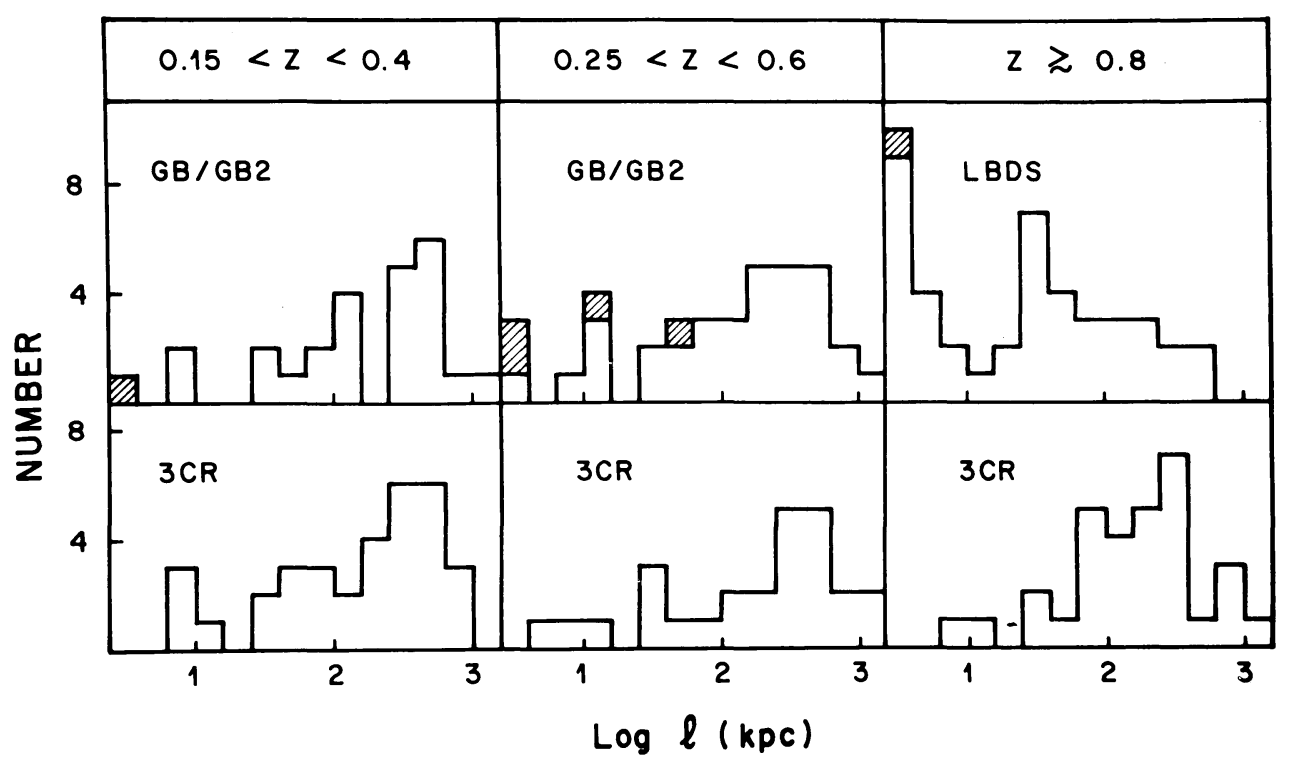

Fig. 6. Comparison of linear size distributions for three samples of radio galaxies discussed in the text with $3 \mathrm{CR}$ sources. Hatched area indicates flat spectrum sources. 
larger linear sizes, the difference in sizes being largest in the highest redshift samples. The distributions are consistent with a weak $\mathrm{P}-\ell$ correlation of the form $\ell \propto \mathrm{P}^{0 \cdot 25 \pm 0 \cdot 1}$, assuming the dependence to be similar at different redshifts. A more accurate determination of the relation between $P$ and $\ell$ requires the determination of many more redshifts for weak radio samples. It is interesting to note that a weak correlation between $\mathrm{P}$ and $\ell$ is already known to exist (Gavazzi \& Perola 1978; Ekers et al. 1981) for nearby $(z<0.1)$ galaxies of lower radio luminosity $\left(\mathrm{P}(1.4) \lesssim 10^{25 \cdot 5} \mathrm{WHz}^{-1}\right.$ ster $\left.{ }^{-1}\right)$.

It is seen from Fig. 6 that the LBDS sample has a much larger fraction of SSC sources than the corresponding 3CR sample. This is likely to be due partly to the difference in the frequencies of the two surveys, as such sources are well known to be much more common in higher frequency surveys. It is also possible that several of these sources are optically faint quasars because most of the SSC sources in strong high frequency surveys are known to be quasars at high redshifts. In fact over $50 \%$ of all sources in the Peacock \& Wall (1981) $2.7 \mathrm{GHz}$ catalogue with known or estimated redshifts $\gtrsim 0.8$ are found to be of the SSC variety and over $60 \%$ of these are identified with quasars (Kapahi et al. 1986).

A comparison of the properties of a complete sample of 67 sources (mostly galaxies) from a $151 \mathrm{MHz} 6 \mathrm{C}$ survey (with $2.2<\mathrm{S}(151 \mathrm{MHz})<4.4 \mathrm{Jy}$ ) with those in the 3CR sample has been reported recently by Eales (1985). Spectroscopic redshifts are available for about $24 \%$ of the sample and have been estimated for the rest either from the optical magnitudes ( $42 \%)$ or from the absence of optical identifications. It is found that on average the $6 \mathrm{C}$ sources have larger redshifts but similar radio luminosities compared to the 3CR sample. The angular sizes of the 6C sources are however considerably smaller than for $3 \mathrm{CR}$. The data provide a statistically significant evidence for the dependence of linear sizes on epoch at constant luminosity ( $n=1.45 \pm 0.4$ for $q_{0}=0.5$ and $n=1.1 . \pm 0.5$ for $q_{0}=0$ ), but none for a correlation between size and luminosity at fixed redshift. A weak correlation between $P$ and $\ell$ cannot however be ruled out by Eales' data as the difference in the mean luminosities of the samples compared is not very large.

It is harder to investigate the $\mathrm{P}-\ell$ relation for quasars alone because of the difficulty of defining complete samples. Not only are identifications with quasars and spectroscopic observations incomplete, particularly in low flux density samples, the larger spread in optical luminosities makes it difficult to estimate redshifts from apparent magnitudes. We assume therefore in what follows that $\mathrm{P}-\ell$ relation for quasars is similar to that for galaxies.

\section{COMPARISON WITH COSMOLOGICAL MODELS AND SIZE EVOLUTION}

The predictions of several cosmological models, including the standard Friedmann type models with $\mathrm{q}_{0}=0$ \& 0.5 , and some 'non-standard' models are compared with the observed $\theta-z$ relation in Figure 7 . The predicted relations have all been normalized to a constant value of $\theta_{m}$ at low redshifts. Apart from the static Euclidean relation which fits the data rather well, all the other models show different amounts of departure from the observed data. Although the 'tired light' model (a non expanding universe in which the redshift arises because photons loose energy with distance; e.g. La Violette 1986) cannot be ruled out by the present data, its prediction does appear to depart from observations 


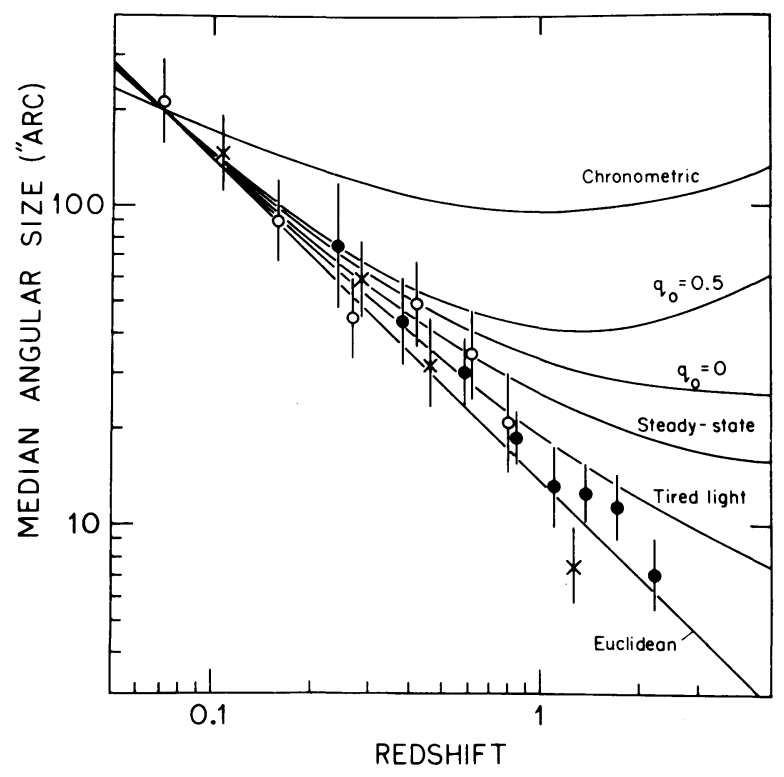

Fig 7. Predictions of several world models compared with the observed $\theta-z$ relation. $\theta_{m}$ values for the 4 samples of galaxies of similar luminosity are shown by crosses. The curves have all been normalized to $\theta_{\mathrm{m}}=200 "$ arc at $\mathrm{z}=0.07$.

at the highest redshifts, particularly with respect to the data for galaxies of constant luminosity. Note that the model with $\mathrm{q}_{0}=0$ has the steepest possible relation between $\theta$ and $z$ among the 'standard' models. The prediction of the Chronometric model (Segal 1976) appears to be grossly discrepant with the observations.

It is clear from Fig.7 that in the Friedmann models the linear sizes must depend more and more strongly on cosmic epoch the larger the value of $\mathrm{q}_{\mathrm{o}}$. But since the nature and amount of evolution is far from being understood at the present state of our knowledge about the formation and evolution of radio sources, the $\theta-z$ relation cannot be used to determine a value of $q_{0}$. It is more meaningful therefore to determine the amount of evolution needed for a given value of $\mathrm{q}_{\mathrm{o}}$ in order to compare with possible models for the physical nature of the evolution.

The amount of evolution with epoch could in general depend on the radio luminosity as well. There is insufficient data however to consider this general case at present. We shall assume that the epoch dependence is the same over the range of luminosity covered by the data. We also assume the simplest mathematical form of epoch dependence, viz. $\ell(z) \propto(1+z)^{-n}$. This refers of course to the maximum linear sizes attained by the populations of radio sources at different epochs and not to the temporal evolution of individual sources because the life times of radio sources are likely to be much smaller than the Hubble time.

The expected $\theta-z$ relations for different values of $n$ in the $q_{0}=0$ and 0.5 models are shown in Figures 8 and 9 respectively. Good fits to the data are obtained for values of $n=1.5 \pm 0.5$ for $q_{0}=0$ and $n=2 \pm 0.5$ for $\mathrm{q}_{\mathrm{o}}=0.5$. The fits shown in the figures correspond to local median linear 

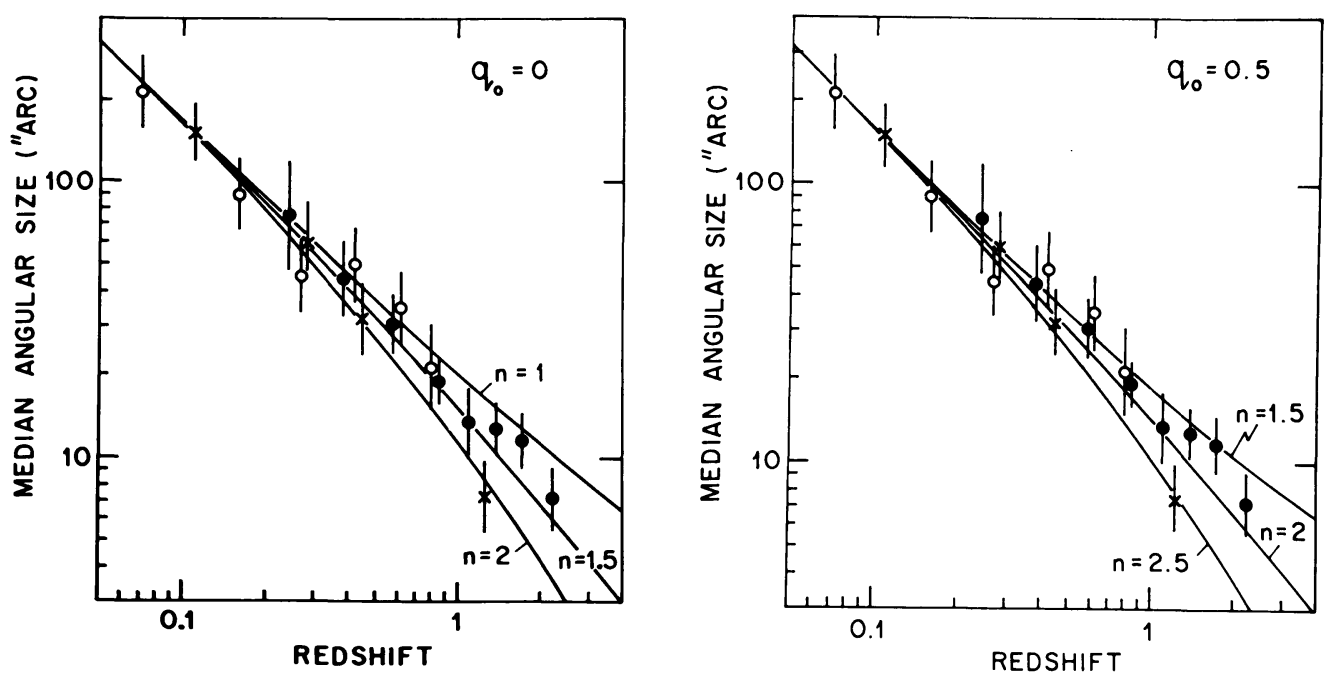

Figs. 8 \& 9. Predicted relations for different amounts of size evolution for $\mathrm{q}_{\mathrm{O}}=0$ and 0.5 respectively.

sizes $2(z=0)$ of about 450 to $475 \mathrm{kpc}$.

In deriving the evolution parameter we have ignored a possible inhomogeneous distribution of matter in the Universe which can have the effect of steepening the $\theta-z$ relation (Roeder 1975). The effect is, however, applicable only for $\mathrm{q}_{\mathrm{o}}>0$ and as noted by Katgert (1977) has a relatively small effect on the determination of $n$ even for $q_{0}=1$ and the extreme assumption that the entire mass density of the Universe is condensed into galaxies.

\section{5. $\theta-z$ RELATION FOR 'HOTSPOTS'.}

The $\theta-z$ test has been applied also to the sizes of hotspots (typically $\lesssim 5 \mathrm{kpc}$ ) generally found at the extremities of the outer lobes of extragalactic sources by estimating their sizes from observations of interplanetary scintillations (IPS) at $81 \mathrm{MHz}$ (Hewish et al. 1974; Readhead \& Hewish 1976). The results were interpreted to imply either an increase in the physical sizes of hotspots with increasing radio luminosity or a rather high value for $q_{0}$ $\left(0.5<\mathrm{q}_{0}<2\right)$. Although hotspots sizes may be less likely to show strong projection effects, the determination of their sizes from IPS observations may suffer from effects of blending of different source components (eg. Swarup \& Bhandari 1976). Several subsequent observations with higher angular resolution (eg. Kapahi \& Schilizzi 1979) have infact found structure in the radio lobes on a scale considerably smaller than the IPS estimates. Synthesis maps of the hotspots in quasars do not appear to show any strong dependence of their physical sizes on redshift or radio luminosity (Swarup et al. 1984). High resolution ( 0.1 to 0.3 arcsec) maps of much larger samples covering a wide range in radio luminosity and redshift are however needed to investigate the $\theta-z$ relation for hotspots reliably. 


\section{THE $\theta-$ S RELATION}

A useful variant of the $\theta-z$ test is the angular size - flux density ( $\theta$ - S) relation, first investigated by Swarup (1975) and Kapahi (1975). This has the advantage that large unbiased samples of radio sources can be used without having to measure redshifts. It is necessary, however, in interpreting the relation to relate flux density to redshift through models for the epoch dependence of the radio luminosity function (RLF) that have been developed in order to fit the extensive source count data and other available information on redshifts, optical identifications, spectral index distributions etc. at various flux levels.

In the last ten years the $\theta$ - S relation has been confirmed and extended to lower flux levels ( $\sim 0.1 \mathrm{Jy}$ at $408 \mathrm{MHz}$ and $\sim 1 \mathrm{mJy}$ at $1.4 \mathrm{GHz}$ ) using high resolution observations of many complete samples (eg. Ekers \& Miley 1977; Downes et al. 1981; Kapahi \& Subrahmanya 1982; Fielden et al. 1983; Windhorst et al. 1985a). For samples selected at or near $408 \mathrm{MHz}$ (where the compact flat-spectrum sources make a negligible contribution at all flux levels) it is now well established that $\theta_{\mathrm{m}}$ reaches a constant value of $\sim 8$ to 10 arcsec at low flux levels (see Fig.10). The observed relation can be compared with predictions (for an assumed world model) based on an evolving RLF and a local size function derived from the observed linear sizes of nearby sources in a well observed sample such as 3CR. On the assumption that the size function is independent of radio luminosity (there is no evidence of a $\mathrm{P}-\ell$ relation among the nearby $3 C R$ sources) the predicted values of $\theta_{\mathrm{m}}$ are found to be considerably higher than observed at lower flux levels and can be made to fit the data only by invoking evolution in linear sizes (Kapahi 1975, 1977; Swarup \& Subrahmanya 1977; Katgert 1977). Narlikar \& Chitre (1977) have attempted to explain the observations without evolution but their claims have been questioned by Subrahmanya (1977).

An improvement over the above method (Downes et al. 1981) is to use each source in a well observed parent sample of bright sources individually to estimate the contribution of sources of that luminosity to different bins of flux density and angular size by a $\mathrm{V} / \mathrm{V}_{\mathrm{m}}$ type of analysis in which the known evolution in the RLF is taken into account. The method assumes that the type of sources found in weaker samples are well represented in the parent sample and any relation between $P$ and $\ell$ that may be present in the parent sample is automatically taken into account. Predictions using this technique and the $3 \mathrm{CR}$ parent sample are also found to fit the data (including distributions of $\theta$ at different flux levels) only when size evolution is included in the computations (Kapahi \& Subrahmanya 1982), contrary to the conclusions of Downes et al. (1981).

The choice of the parent sample in predicting $\theta$-S relations has also created some controversy. It was pointed out by Downes (1982) that if week sources in low frequency surveys have large redshifts the observed emission would correspond to much higher emitted frequencies so that the observed $\theta_{m}$ values may be too low because of the presence of a large number of steep-spectrum compact (SSC) sources which constitute a substantial fraction of the sources found in high frequency surveys. Parent samples selected at high frequencies may therefore be more appropriate. Predictions based on the Peacock \& Wall catalogue of strong sources at $2.7 \mathrm{GHz}$ and the multifrequency evolutionary models of the RLF investigated by Peacock \& Gull 
(1981) have led Downes (1982), Fielden et al. (1983) and Allington-Smith (1984) to conclude that in at least one or two RLF models the observed ${ }^{\theta} \mathrm{m}$ values at low flux densities at $408 \mathrm{MHz}$ can be explained without need for any evolution in linear sizes. This has been examined recently by Kapahi et al. (1986), who point out that predictions based on high frequency surveys overestimate the contribution of SSC sources to low frequency samples at all flux levels because models of the evolving RLF do not take into account the low frequency curvature in the spectra of such sources. Because SSC sources occur at fairly high redshifts even in bright source samples at high frequencies the use of such samples to predict $\theta_{\mathrm{m}}$ values in deep low frequency samples is inappropriate unless the latter have extremely high redshift of $\approx 10$. Furthermore, recent high resolution observations of weak 5C sources do not show an abnormally large number of SSC sources (Goapl-Krishna et al. 1986). By correcting partially for the spectral curvature in SSC sources, new predictions based on parent samples selected at 1.4 and $2.7 \mathrm{GHz}$ and a wide range of possible evolutionary schemes for the RLF suggested in the literature (Peacock \& Gull 1981; Subrahmanya \& Kapahi 1983; Condon 1984) it is found (Kapahi et al. 1986) that the $\theta$ - S data cannot be fitted without invoking evolution in linear sizes, even for $\mathrm{q}_{\mathrm{o}}=0$. Predictions based on the $2.7 \mathrm{GHz}$ sample are reproduced in Fig.10. Values of $\mathrm{n}$ between about 1 and 2.5 are indicated depending on $q_{o}$, the parent sample

PARENT SAMPLE - PW $(2.7 \mathrm{GHz})$
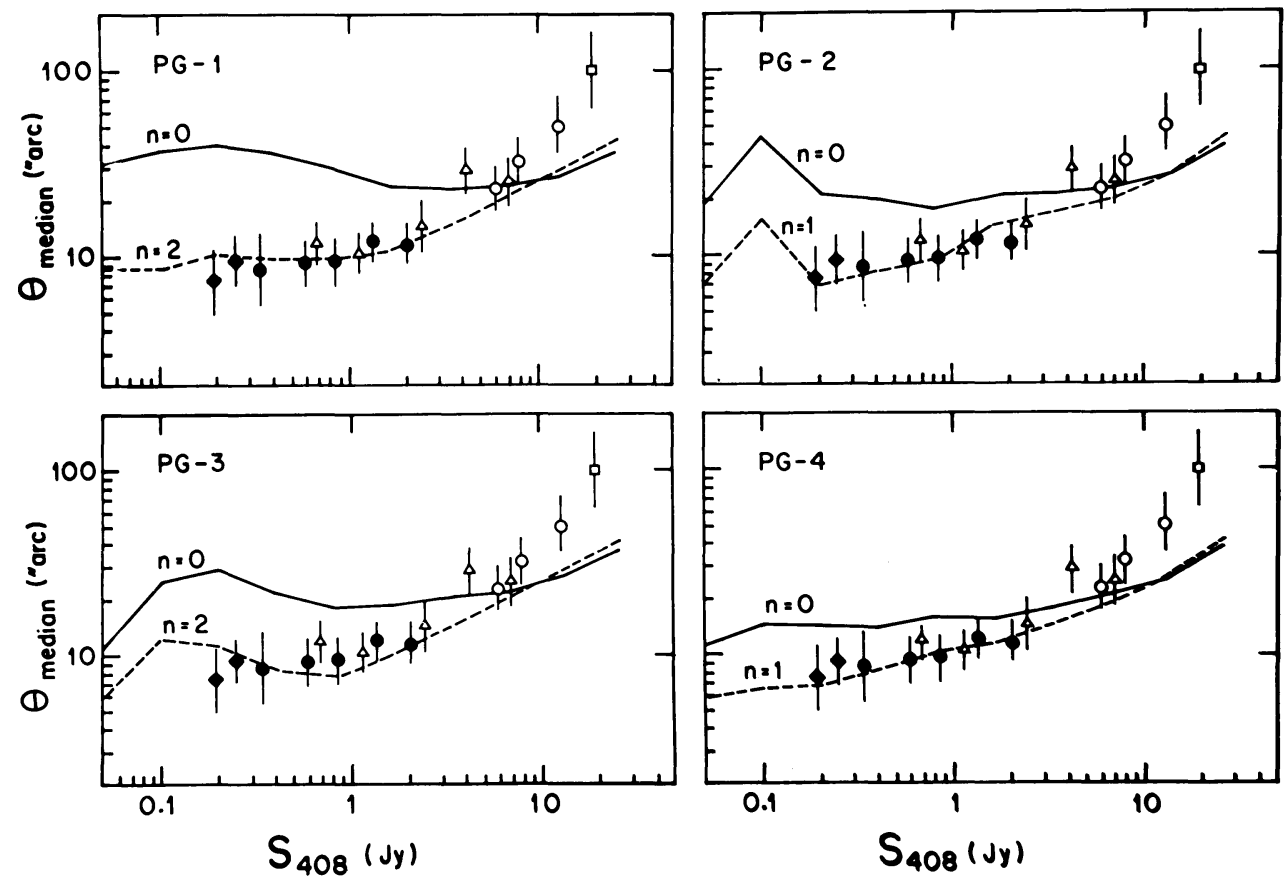

Fig. 10. Predicted $\theta-\mathrm{S}$ relations for the $2.7 \mathrm{GHz}$ parent sample and the four evolutionary RLF models of Peacock \& Gull (1981), with and without size evolution. RLF models la \& 2 are for $\mathrm{q}_{\mathrm{o}}=0.5$ and $3 \& 4$ for $\mathrm{q}_{\mathrm{o}}=0$. 
and the evolutionary model. This is in good agreement with the evolution inferred from the $\theta-z$ relation discussed earlier.

From a high resolution deep VLA survey, Coleman \& Condon (1985) have recently extended the $\theta$ - S relation to extremely low flux levels of $\sim 0.1 \mathrm{mJy}$ at $1400 \mathrm{MHz}$. They find that the value of $\theta_{\mathrm{m}}$ drops again below $\sim 1 \mathrm{mJy}$, reaching $\lesssim 3$ arcsec at the lowest flux levels. They interpret this as arising from the faintest sources residing in spiral or Seyfert galaxies unlike the stronger ones which are produced largely by elliptical galaxies. Using a simple scheme based on the BDFL parent sample and the evolutionary model of Condon (1984) they have fitted the entire $\theta$ - S relation without requiring size evolution. While the change in population from elliptical to spiral galaxies may indeed be responsible for the fall off in $\theta_{m}$ at very low flux levels, their conclusion about not requiring size evolution is, however, misleading because their scheme for predicting the $\theta$ - S relation is over simplified. Instead of treating each source in the BDFL sample individually in order to estimate its contribution to different flux density and angular size bins they have summed the number of BDFL sources in different ranges of linear size (as calculated from their redshifts and angular sizes) to obtain a distribution of intrinsic sizes assumed to be independent of radio luminosity. But since many sources in BDFL are seen at high redshifts, the effect of evolution in linear sizes is already present in the derived size distribution which is then used to predict values of $\theta_{\mathrm{m}}$ at different flux levels.

\section{WHAT CAUSES SIZE EVOLUTION?}

Two possible causes for the evolution of linear sizes have been in the literature for quite some time, namely the increase with epoch in the energy density of the microwave background radiation and in the density of an intergalactic medium.

The increased radiative losses at high redshifts due to inverse Compton scattering off the microwave background photons can snuff out radio sources (Rees \& Setti 1968) before they have reached large physical sizes. Unless the magnetic fields in radio sources are much weaker than implied by equipartition arguements, however, inverse Comption losses do not appear to dominate over synchrotron losses even at fairly high redshifts (Scheuer 1977). The importance of the inverse Compton losses has been investigated by Allington-Smith (1984) and Eales (1985), who find that their effect on linear size evolution, for realistic models of radio sources, is rather small, although it may be important for some of the largest sources. The similar range in observed angular sizes at different redshifts (Figures 1 and 2), also argues against this effect being important in determining the distribution of linear sizes.

The ram pressure confinement of radic sources due to an intergalactic medium, whose cosmological density should vary as $(1+z)^{3}$, can slow down the expansion of the hot spots so that sources would attain smaller physical sizes at earlier epochs, assuming that the activity in their central engines lasts for a fixed period of time. But the existence of an intergalactic medium of sufficient density is uncertain. Two recent developments are however noteworthy. First, analysis of X-ray data from the Einstein Observatory has indicated (Forman et al. 1985) that hot gaseous coronae (extending to 
a radius of $\sim 100 \mathrm{kpc}$ in some cases) are perhaps an ubiquitous feature of bright early type galaxies, including isolated ellipticals. Second, the possibility that the 5-200 kev X-ray background arises from a hot intergalactic medium has been revived recently by Guilbert \& Fabian (1986). The propagation of the twin beams of radio sources as they plough their way first through the hot coronae of the parent galaxies and then through the hot intergalactic medium has been investigated recently by Gopal-Krishna \& Wiita (1986), by assuming that the two media are pressure matched at their interface which moves closer to the parent galaxy at higher redshifts due to the rising pressure of the intergalactic medium. This hastens the onset of rapid deceleration of the beam heads whose advance velocity becomes subsonic relative to the IGM at earlier times at higher redshifts, and the growth of linear size can subsequently be ignored inspite of continuing nuclear activity. Their model appears to provide a possible explanation for the strong evolution of linear sizes with epoch.

The evolution of galactic haloes with epoch is at present unknown. Even on the conservative assumption that galactic halos do not evolve significantly with epoch, the interface between them and the IGM would be expected to take place close to or within the confines of the parent galaxy at high redshifts $(z \geq 2)$. This may be responsible (Gopal-Krishna \& Wiita 1986) for the subgalactic dimensions of a large number of quasars (SSC sources) and for the observed increase in distorted radio structures at high redshifts (Barthel 1986; see also Miley's contribution in the present symposium) which may be caused by a substantial heating of and the consequent bulk flows of the interstellar gas due to the discharge of large amounts of momentum flux by the twin beams in such small volumes (e.g. Lonsdale \& Barthel 1986).

\section{CONCLUSION}

The angular size - redshift relation is important for observational cosmology as it provides valueable information on th evolution with epoch of the linear sizes of extragalactic radio sources and can be used for discriminating against some models of the Universe. The physics of the evolution of individual sources and the various factors responsible for the inferred evolution with epoch are, however, far from being understood. There is therefore little hope at this stage of using the relation as a test of the geometry of the Universe.

\section{REFERENCES}

Allington-Smith, J.R. 1984, M.N.R.A.S., 210, 611.

Barthel, P.D. 1986, in Quasars, IAU Symp.119, eds. G.Swarup \& V.K.Kapahi, D.Reidel, Dordrecht, p.181.

Bridle, A.H., Davis, M.M., Fomalont, E.B. \& Lequeux, J. 1972, A.J., 77, 405. Bruzual, G.A. \& Spinrad, H. 1978, Ap.J., 220, 1.

Coleman, P.H. \& Condon, J.J. 1985, A.J., 90, 1431.

Condon, J.J. 1984, Ap.J., 287, 461.

Downes, A.J.B. 1982, in Extragalactic Radio Sources, IAU Symp. 97, eds. D.S.Heeschen \& C.M.Wade, D.Reidel, Dordrecht, p.393. 
Downes, A.J.B., Longair, M.S. \& Perryman, M.A.C. 1981, M.N.R.A.S., 197, 593.

Downes, A.J.B., Peacock, J.A., Savage, A. \& Carrie, D.R. 1986, M.N.R.A.S., 218,31 .

Eales, S.A. 1985, M.N.R.A.S., 217, 179.

Ekers, R.D. \& Miley, G.K. 1977, in Radio Astronomy and Cosmology, IAU

Symp.74., ed. D.L.Jauncey, D.Reidel, Dordrecht, p.109.

Ekers, R.D., Fanti, R., Lari, C. \& Parma, P. 1981, A \& A., 101, 194.

Fanaroff, B.L. \& Riley, J.M. 1974, M.N.R.A.S., 167, 31P.

Fielden, J., Downes, A.J.B., Allington-Smith, J.R., Benn, C.R., Longair, M.S. \& Perryman, M.A.C. 1983, M.N.R.A.S., 204, 289.

Forman, W., Jones, C. \& Tucker, W. 1985, Ap.J., 293, 102.

Gavazzi, G. \& Perola, G.C. 1978, A \& A., 66, 407.

Gopal-Krishna, Saripalli, L., Saikia, D.J. \& Sramek, R.A. 1986, in Quasars, IAU Symp.119, eds. G.Swarup \& V.K.Kapahi, D.Reidel, Dordrecht, p.193.

Gopal-Krishna \& Wiita, P.J. 1986, submitted to M.N.R.A.S.

Guilbert, P.W. \& Fabian, A.C. 1986, M.N.R.A.S., 220, 439.

Hewish, A., Readhead, A.C.S. \& Duffett-Smith, P.J. 1974, Nature, 252, 657. Hickson, P. 1977, Ap.J., 217, 964.

Hickson, P. \& Adams, P.J. 1979, Ap.J., 234, L91.

Hooley, T.A., Longair, M.S. \& Riley, J.M. 1978, M.N.R.A.S., 182, 127.

Hoyle, F. 1959, in Paris Symp. on Radio Astronomy, ed. R.N.Bracewell, Stanford Univ. Press, Stanford, p.529.

Jackson, J.C. 1973, M.N.R.A.S., 162, 11 P.

Kapahi, V.K. 1975, M.N.R.A.S., 172, 513.

Kapahi, V.K. 1977, in Radio Astronomy and Cosmology, IAU Symp.74, ed. ed. D.L.Jauncey, D.Reidel, Dordrecht, p.119.

Kapahi, V.K. 1981, A. \& A. Suppl., 43, 381.

Kapahi, V.K. 1985, M.N.R.A.S., 214, $19 \mathrm{P}$.

Kapahi, V.K. 1986, in Highlights of Astronomy, Vol 7, ed. J.P.Swings, D.Reidel, Dordrecht, p.371.

Kapahi, V.K., Kulkarni, V.K. \& Subrahmanya, C.R. 1986, submitted to J.Astrophys. Astron.

Kapahi, V.K. \& Schilizzi, R.T. 1979, Nature, 277, 610.

Kapahi, V.K. \& Subrahmanya, C.R. 1982, in Extragalactic Radio Sources,

IAU Symp. 97, eds. D.S.Heeschen \& C.M.Wade, D.Reidel, Dordrecht, p.401.

Katgert, P. 1977, Ph.D. Thesis, University of Leiden

La Violette, P.A. 1986, Ap.J., 301, 544.

Laing, R.A., Riley, J.M. \& Longair, M.S. 1983, M.N.R.A.S., 204, 151.

Legg, T.H. 1970, Nature, 226, 65.

Lonsdale, C.J. \& Barthel, P.D. 1986, Ap.J., 303, 617.

Machalski, J. \& Condon, J.J. 1985, A.J., 90, 973.

Macklin, J.T. 1982, M.N.R.A.S., 199, 1119.

Masson, C.R. 1980, Ap.3.., 242, 8.

Miley, G.K. 1971, M.N.R.A.S., 152, 477.

Narlikar, J.V. \& Chitre, S.M. 1977, M.N.R.A.S., 180, 525 and 181, 799.

Peacock, J.A. \& Gull, S.F. 1981, M.N.R.A.S., 196, 611.

Peacock, J.A. \& Wall, J.V. 1981, M.N.R.A.S., 194, 331.

Peacock, J.A. \& Wall, J.V. 1982, M.N.R.A.S.g 198, 843.

Readhead, A.C.S. \& Hewish, A. 1976, M.N.R.A.S., 176, 571.

Rees, M.J. \& Setti, G. 1968, Nature, 219, 127.

Roeder, R.C. 1975, Nature, 255, 124. 
Saikia, D.J. \& Kulkarni, V.K. 1979, M.N.R.A.S., 189, 393

Sandage, A. 1961, Ap.J., 133, 355.

Scheuer, P.A.G. 1977, in Radio Astronomy \& Cosmology, IAU Symp.74, ed. D.L.Jauncey, D.Reidel, Dordrecht, p.343.

Segal, I.E. 1976, Mathematical Cosmology and Extragalactic Astronomy, Academic Press, New York.

Stannard, D. \& Neal, D.S. 1977, M.N.R.A.S., 179, 719.

Subrahmanya, C.R. 1977, Ph.D. Thesis, University of Bombay.

Subrahmanya, C.R. \& Kapahi, V.K. 1983, in Early Evolution of the Universe and Its Present Structure, IAU Symp. 104, eds. G.Abell \& G.Chincarini, D.Reidel, Dordrecht, p.47.

Swarup, G. 1975, M.N.R.A.S., 172, 501.

Swarup, G. \& Bhandari, S.M. 1976, Astrophys. Lett., 17, 31.

Swarup, G., Sinha, R.P. \& Hilldrup, K. 1984, M.N.R.A.S., 208, 813.

Swarup, G. \& Subrahmanya, C.R. 1977, in Radio Astronomy \& Cosmology,

IAU Symp.74, ed. D.L.Jauncey, D.Reidel, Dordrecht, p.125.

van Breugel, W., Miley, G. \& Heckman, T. 1984, A.J., 89, 5.

Veron-Cetty, M.P. \& Veron, P. 1985, E.S.O. Scientific Report No.4.

Wardle, J.F.C. \& Miley, G.K. 1974, A. \& A., 30, 305.

Wardle, J.F.C. \& Potash, R. 1977, Ann.N.Y.Acad.Sci., 302, 605.

Wills, D. 1979, Ap.J.Suppl., 39, 291 .

Windhorst, R.A. 1986, in Highlights of Astronomy, Vol.7, ed. J.P.Swings, D.Reidel, Dordrecht, p.355.

Windhorst, R.A., van Heerde, G.M. \& Katgert, P. 1985a, A. \& A. Suppl., 58,1 .

Windhorst, R.A., Kron, R.G. \& Koo, D.C. 1985b, A. \& A. Suppl., 58, 39.

\section{DISCUSSION}

NARLIKAR: I have two comments (1) For the $\theta-z$ plot at high redshifts you took a tentative value of 1.5 for redshift since there were no identifications in your sample $(z>0.8)$. Taking this tentativeness into account the steady state model appears consistent with your $\theta-z$ plot. (2) The non-eve?.ving luminosity function computed by DasGupta et al. was used by DasGupta and me together with weak-inverse correlation between size and power in your luminosity range $\log \mathrm{L}$ between 24.5 to 26 to reproduce your $\theta-z$ plot. Whether such inverse correlation exists can be decided by looking at large samples of radio sources which are nearby (to avoid confusion with evolution).

KAPAHI: (1) Although there is indeed some uncertainty about the median redshift of the deep LBDS sample $\left(z_{m} \sim 1\right.$ to 3 ?), this is not crucial to my argument. The observed median size for this sample cannot in fact be made to fill the steady-state prediction whatever the actual vaiue of $z_{\mathrm{m}}$ is for this sample. (2) In this luminosity range there is just no evidence in the data for an inverse relation between size and power. The data in fact indicate a weak direct correlation between the two. 
WALSH: $n_{n}$ vou 11se the same evolution factor for compact steep-spectrum sources as for more extended ones? There is some evidence that they are qualitatively different: possibly the SSC sources have not burst out of their parent galaxy whereas the more extended ones have, so that they may evolve in different ways.

KAPAHI: Yes, models of the evolving RLF do not distinguish between steep-spectrum compact (SSC) sources and more extended ones as far as evolution is concerned. The data on quasars shows quite clearly that SSC sources are generally of high radio luminosity and are much more likely to be at high redshifts, which is consistent with strong evolution.

MACHALSKI: Do the medians shown contain sources larger than $20 \mathrm{Kpc}$, or all sources including steep spectrum compact sources? I am not sure that using medians is the best method to investigate any distribution since one can imagine completely different distributions with the same median. A non-parametric test would be much more appropriate for this purpose.

KAPAHI: Sources with $\ell<20 \mathrm{Kpc}\left(\mathrm{q}_{\mathrm{O}}=\frac{1}{2}, \mathrm{H}_{2}=50\right)$ have indeed been excluded from the $\theta-z$ plots in estimating values of $\theta_{\text {median }}$ But as I pointed out, including them would only strengthenetian case for size evolution. The observed distributions of log $\theta$ are quite well behaved and median values are simple to estimate. The estimated value of $\theta_{m}$ and the errors are indeed non-parametric because no particular form for the distribution of $\Theta$ is assumed. 